\title{
Unveiling of Taste Disorders: An Overview
}

\author{
${ }^{1}$ Aparna Srivastava, ${ }^{2}$ Poornima Govindraju, ${ }^{3}$ Balaji Pachipulusu
}

\begin{abstract}
Taste is the sensation produced when a substance in the mouth react chemically with the taste receptor cells located on the taste buds. Taste abnormality associated with abnormal substances, such as food debris, dental plaque, abnormal salivary constituents, metabolic products of bacteria and fungi and inflammatory exudates in the oral cavity. Accurate diagnosis of taste loss is the foremost step in the treatment of taste abnormalities. It is therefore helpful for both patient and health care provider to unravel the accurate cause.
\end{abstract}

Keywords: Diagnosis, Dysgeusia, Taste, Taste disorder, Tongue. How to cite this article: Srivastava A, Govindraju P, Pachipulusu B. Unveiling of Taste Disorders: An Overview. J Health Sci Res 2016;7(1):12-15.

Source of support: Nil

Conflict of interest: None

\section{INTRODUCTION}

Taste is the sensation produced when a substance in the mouth reacts chemically with the taste receptor cells located on the taste buds. The sense of taste delivers vital sensory information to determine whether a particular food or beverage will be ingested. Taste disturbances can array from a total loss of taste to the constant occurrence of phantom tastes, such as a bitter or metallic taste in the absence of any offending substance in the mouth. It is significant to identify taste impairment, even if it is not practical to measure them. These symptoms have real-world bases that while not necessarily similar with the specifics of the patient complaint, can impact significantly on quality of life. Exact diagnosis of taste loss is the foremost step in the treatment of taste abnormalities. It is, therefore, helpful for both patient and health care provider to be aware of the causes of taste changes. Proving the etiology of a dysgeusia is not an easy matter because of the number of anatomical and functional structures involved, the multidisciplinary nature of the etiologies, and the extreme difficulty of dealing with a rare condition.

\footnotetext{
${ }^{1}$ Postgraduate Student, ${ }^{2}$ Reader, ${ }^{3}$ Professor and Head

${ }^{1-3}$ Department of Oral Medicine and Radiology, RajaRajeswari Dental College and Hospital, Bengaluru, Karnataka, India
}

Corresponding Author: Aparna Srivastava, Postgraduate Student, Department of Oral Medicine and Radiology RajaRajeswari Dental College and Hospital, Bengaluru Karnataka, India, Phone: +919008421514, e-mail: dentistaparna 04@gmail.com
A thorough knowledge of the anatomy and physiology of the gustative system associated with an elaborate anamnesis and a thorough examination of the oral cavity are the solutions. ${ }^{1}$ The rudimentary step to diagnose taste disorders is to start with patient's medical history.

\section{PATIENT'S MEDICAL HISTORY}

Most essential to identify is whether patients can distinguish salt, sour, sweet, and bitter. It is important to ascertain whether the reported taste disturbance was of acute onset or was gradual in nature. Acute taste loss is often associated with iatrogenic or toxic causes, whereas a more insidious history may suggest an underlying neurological or neoplastic process. Any concomitant loss of olfactory function should also be ascertained. A full medical history, including use of any medications, when they were started, and how this relates to the onset of the taste disorder, is essential. Assessment of existing medical conditions, a history of any precipitating event, such as recent trauma, medical procedure, or radiotherapy, is also recommended. ${ }^{2}$ Once, thorough history is taken complete examination needs to be done.

\section{PHYSICAL EXAMINATION}

The number, size, and distribution of papillae on the surface of the tongue should be examined; topical anesthesia is applied to the tongue to diagnose persistent dysgeusia after anesthesia in case of phantom taste; thermal stimulation is to test persistent cold sensation in the area of the foliate papillae in case of glossopharyngeal nerve integrity. ${ }^{1}$ Quantity and quality of saliva should be examined, and condition of teeth should be noted. Depending on the clinical history, a neurological examination, including assessment of cognitive function, is indicated. This should include a careful evaluation of cranial nerve function. ${ }^{3}$

\section{OBJECTIVE EXAMINATION}

Taste disorders are a result of their burdensome nature; objective examinations will be reserved for patients for whom the clinical and gustometric tests do not permit the establishment of etiology. Culture is suggested when a fungal or a bacterial buccal infection is suspected. Sialometry and salivary biopsy to exclude salivary dysfunction. The use of imaging depends on the diagnostic hypothesis. Computed tomography (CT) is appropriate when there is a suspected posttraumatic fracture or erosion 
of bone in the area of the middle ear. Magnetic resonance imaging (MRI) is the examination of choice when evaluating the cortical structures and the pons in search of an ischemic, hemorrhagic, demyelinating, tumorous, or epileptic pathology. A blood test can also be preferred and it will focus on the blood count (anemia, effects of medication). ${ }^{3}$

Investigations for taste disorders are:

- Simple taste tests

- Blind taste test

- Methylene blue staining

- Topical anesthesia test

- Intensity test

- Hedonic scale

- Specialized taste tests

- Chemosensory gustatory testing

- Electrogustometer

- Tasters

- Taste sensor

- Others

- CT/MRI

- Video micrography

- Isotope technique

- Direct microscopic examination.

\section{SIMPLE TEST}

\section{Blind Taste Test}

It is an easiest test to perform for the recognition of the taste. Mask the eyes of patient, use four taste solution (salt, sour, bitter, and sweet), and apply it on the dorsal surface of tongue. Ask the patient to recognize the taste. Repeat the same procedure for the next solution. ${ }^{4}$

\section{Methylene Blue Staining of the Tongue}

This is used to test gross innervation of taste buds. Taste pores remain stained (blue) if they are innervated. Taste pores do not remain stained if there is an interruption of innervation. ${ }^{5}$

\section{Topical Anesthesia applied to the Tongue}

Topical anesthesia distinguishes between oral and nonoral sources of dysgeusia. Apply $2 \%$ unflavored viscous lidocaine or $1 \%$ dyclonine hydrochloride to four quadrants of the tongue in a sequential fashion (left anterior 2/3, left posterior $1 / 3$, right anterior $2 / 3$, right posterior $1 / 3$ ) with a cotton-tipped applicator. Total mouth rinse with topical anesthetic to anesthetize taste buds located in the anterior portion of the oropharynx and hard and soft palates. Caution patients about a reduced gag reflex after application of anesthetics. Advise patients to avoid eating and drinking until anesthetics have worn off. If dysgeusia is reduced, cause may be local and if dysgeusia is greater, cause may be central (e.g., phantom taste). If no change in dysgeusia, cause is probably not oral. ${ }^{6}$

\section{Intensity Test}

To test the sense of taste, strong solution of sugar (for sweet), common salt (for salt), weak solution of citric acid (for sour), and quinine (for bitter) are used. Four vials of solutions are placed on the table with labels, dry the tongue with paper towel, and apply the different solutions with applicator stick/glass rod to the tip, sides, and back of the tongue as follows:

Solution of $5 \%$ sucrose, $1 \%$ acetic acid, $5 \% \mathrm{NaCl}$, and $0.5 \%$ quinine sulfate are prepared.

\section{Sweet Solution}

Mix 2 teaspoons $(\sim 8 \mathrm{gm})$ of sugar in 1 glass $(\sim 250 \mathrm{~mL})$ of water.

\section{Salt Solution}

Mix 3 teaspoons ( 15 gm) of salt in 1 glass $(\sim 250 \mathrm{~mL})$ of water.

\section{Sour Solution}

It usually takes 5 to 10 teaspoons of lemon juice per glass to get the solution to have the same "taste intensity" as salt solution.

\section{Bitter Solution}

Mix 2 teaspoons ( $114 \mathrm{mg}$ caffeine) of instant coffee in 1 glass $(\sim 250 \mathrm{~mL})$ of water.

\section{Procedure}

Inform the volunteer that you will be testing different tastes and asking him/her to rate where the taste feels stronger. Ask the volunteer to rinse his/her mouth with a sip of water, occlude both his/her nostrils, and stick his/her tongue out. Dip a clean cotton swab into the first solution and place it on the tip of the volunteer's tongue. Soak the cotton swab well to get a big drop and allow a few seconds for the tastant to reach the taste buds. Dip the cotton swab into the same solution again and touch the left side. Repeat for the right side and the back of the tongue, each time dipping the cotton swab in the solution again. Ask the volunteer to rate the strength of each taste (on a scale from 1 to 4 , four being the strongest of all). ${ }^{4}$

\section{Hedonic Scale}

Common scales used in testing. These are of two types:

1. Category scales: It is one of the ancient method of scaling and involves the choice of discrete response alternatives to denote increasing sensation intensity in terms of liking and/or preference. The most popular category scale used in sensory testing is hedonic scale which measures the amount of like 
or dislike for the sensory characteristics of food due to their easiness, and category scales are well suited for consumer panel. Moreover, some advantages in data coding and tabulation they offer (for speech and accuracy) because they are easier to tabulate than line markings or the more variable magnitude estimates.

2. Line scales: Line scales may also be denoted to as graphic ratings or visual analog scales with line scales, and participant's reaction can be recorded. ${ }^{7}$

\section{SPECIALIZED TEST}

\section{Chemosensory Gustatory Testing}

Chemical gustometry is a rapid and nonexpensive test. Solutions in increasing concentrations of citric acid, glucose, sodium chloride, and quinine are applied to the tongue with the aid of a dropper or taste strips. ${ }^{8} \mathrm{Chemical}$ gustometry tests the functioning of the receptor cells for each of the archetypical tastes. It is particularly useful in detecting dissociated dysgeusia. Although rather indefinite in terms of determining the exact extent of the enthused zone, it can nevertheless provide topographical information about the taste receptors: Quinine is preferentially recognized in the circumvallate papillae and the foliate papillae, the innervation of which depends on the glossopharyngeal nerve; citric acid, glucose, and sodium chloride are recognized in the anterior portion of the tongue, the innervation of which depends on the chorda tympani. In case of a nerve lesion, the identification of the thresholds will be overvalued within the confines of the topography of innervation. ${ }^{9}$

\section{Electrogustometry}

It is a fast and simple test. Electro-anodic stimulation with unremitting current causes hydrolysis of the saliva, the resultant liberation of ions stimulates the gustative chemoreceptors..$^{10}$ The intensity of the stimulation is amplified until a metallic sensation is detected which states a threshold of detection which is illustrative of the overall function of the taste buds and gustative nerve paths and therefore, helps to discriminate between ageusia and hypogeusia. When this test is used in the different zones of the oral cavity, the advantage is that the precise point of stimulation is known and the affected nerve is located quickly. ${ }^{5}$ Four points from Gomez's study, which included a large number of normal patients $(n=147)$, are worth keeping in mind when interpreting the results:

- The thresholds on the right side can be lower than those on the left and it is because of the specialization of the right-hand hemisphere of the brain in matters of taste perception.
- A difference between the thresholds on the two sides of $19.5 \mu \mathrm{A} \pm 4.5 \mu \mathrm{A}$ for an average threshold of $38.5 \mu \mathrm{A} \pm 5.5 \mu \mathrm{A}$ is permissible.

- A threshold difference of at least $50 \%$ from one side to the other is considered pathological.

- The threshold is higher in the region of the lingual vestibule than in the region of the tongue or the soft palate.

A threshold difference of at least $50 \%$ from one side to the other is considered pathological. ${ }^{11}$

\section{Tasters}

The flavor of peppermints is an auxiliary of PROP (6-n-propylthiouracil), which is a genetic marker for taste and is used in measuring taste sensitivity, because peppermint flavor is more pleasurable and measures more aspects of flavor. The response to peppermint comprises not only taste (for the sweetness), but also smell and sensitivity to trigeminal stimulation (the cool feeling of mint). Most foods present some combination of these sensations, so testing with peppermints reflects general flavor sensitivity, the intensity of the "rush" of sensation people may get in the back of the throat and the nose as well as the intensity of flavor. This rush of sensation is due to the activation of the trigeminal nerve endings in the taste buds and in the nose: Sucrose for sweet sensitivity, citric acid for sour sensitivity, and of course sodium chloride for salt sensitivity. ${ }^{3}$

\section{Taste Sensors}

The five basic taste plays an important role for humans. Saltiness, which is instigated mainly by ionic materials, acts as a noble indicator of electrolyte balance in foods; sourness, which is produced by organic acids, signals decomposition; bitterness, which is often considered distasteful, prevents intake of poisonous materials; umami, which is induced by some chemicals like amino acids that provides information on the presence of amino acids; sweetness, which is produced by sugars or sugar alcohols, has a role in representing nutrient sources. ${ }^{12-14}$ Astringency, which is produced mainly by tannins, is sometimes considered a taste quality in the broad sense. The "fluid mosaic model" was proposed to explain the structure of biological membranes in the early 1970s. ${ }^{15}$ In this model, proteins move in a sea of lipid molecules on cell membranes, including taste cells. Recent advancements have identified the taste receptor cells on the human tongue for the five basic tastes; their signal pathways using artificial lipid-based membrane. ${ }^{16}$

\section{OTHER DIAGNOSTIC TEST}

Cineradiographic studies are beneficial to study the oral cavity and pharynx during drinking, chewing, suckling, 
and phonation and it helps in understanding the shape, position, and movement of the tongue during these activities and also the congenital surgical defects. In addition, $\mathrm{CT} / \mathrm{MRI}$ is beneficial to identify space occupying lesions and muscle atrophy secondary to deep-seated lesions in the base of the tongue. Doppler ultrasound (pulsed) is used to study any conceded blood flow in lingual arteries and other branches of the carotid. The ventral surface of the tongue can be studied by real time (grey scale, B mode) ultrasound to distinguish a solid and vascular lesion from a fluid-filled cavity, such as cyst or abscess. Direct microscopic examination is useful to study capillary blood flow on the dorsum of the tongue, changes in taste papillae, and decreased blood flow in the tongue. Video microscopy and stereomicroscopy are useful to envisage taste pores, papillae, and their capillary network. ${ }^{4,5}$

\section{CONCLUSION}

The number of anatomical and functional structures involved and the multidisciplinary nature of the etiologies cause extreme difficulty in dealing with the taste disorders, so a sound knowledge of the anatomy and physiology of the gustative system is required and thorough investigation may lead to the exact diagnosis and also provide accurate treatment of taste disorders.

\section{REFERENCES}

1. Collet S, Rombaux P, Eloy P, Bertrand B. Taste disorders. B-ENT 2007;3(6):71-79.

2. Pribitkin E, Rosenthal MD, Cowart BJ. Prevalence and causes of severe taste loss in a chemosensory clinic population. Ann Otol Rhinol Laryngol 2003;112:971-978.

3. Osaki T, Ohshima M, Tomita Y, Matsugi N, Nomura Y. Clinical and physiological investigations in patients with taste abnormality. J Oral Pathol Med 1996 Jan;25(1):38-43.
4. Cullen MM, Leopold DA. Disorders of smell and taste. Med Clin North Am 1999 Jan;83(1):57-74.

5. Seiden AM, Duncan HJ, Smith DV. Office management of taste and smell disorders. Otolaryngol Clin North Am 1992 Aug;25(4):817-835.

6. Kuga M, Ikeda M. Evaluation of gustatory threshold changes in healthy subject. Nihon Jibiinkoka Gakkai Kaiho 1996 Mar;99(3):411-416.

7. Lim J. Hedonic scaling: a review of methods and theory. Food Qual Prefer 2011 Dec;22(8):733-747.

8. Mueller C, Kallert S, Renner B, Stiassny K, Temmel AF, Hummel T, Kobal G. Quantitative assessment of gustatory function in a clinical context using impregnated "taste strips". Rhinology 2003 Mar;41(1):2-6.

9. Shingai T, Beidler LM. Response characteristics of the three taste nerves in mice. Brain Res 1985 Jun;335(2):245-249.

10. Tomita H, Ikeda M. Clinical use of electrogustometry: strengths and limitations. Acta Otolaryngol (Suppl) 2002;546: 27-38.

11. Bergdahl M, Bergdahl J. Perceived taste disturbance in adults: prevalence and association with oral and psychological factors and medication. Clin Oral Investig 2002 Sep;6(3): 145-149.

12. Kobayashi Y, Habara M, Ikezazki H, Naito Y, Toko K. Advanced taste sensors based on artificial lipids with global selectivity to basic taste qualities and high correlation to sensory scores. Sensors 2010 Apr;10(4):3411-3443.

13. Legin A, Rudnitskaya A, Vlasov $Y$, Di Natale C, Davide F, D'Amico A. Tasting of beverages using an electronic tongue. Sens Actuat B: Chem 1997 Oct;44(1-3):291-296.

14. Vlasov $Y$, Legin A. Non-selective chemical sensors in analytical chemistry from "electronic nose" to "electronic tongue". Fresenius J Anal Chem 1998 Jun;361(3):255-260.

15. Legin A, Rudnitskaya A, Vlasov $Y$, Di Natale C, Mazzone E, D'Amico A. Application of electronic tongue for qualitative and quantitative analysis of mineral water and wine. Electroanalysis 1999 Jul;11(10-11):814-820.

16. Di Natale C, Paolesse R, Macagnano A, Mantini A, D'Amico A, Ubigli M, Legin A, Lvova L, Rudnitskaya A, Vlasov Y. Application of a combined artificial olfaction and taste system to the quantification of relevant compounds in red wine. Sens Actuat B: Chem 2000 Oct;69(3):342-347. 